\title{
Identification of Profound Metabolic Alterations in Human Dendritic Cells by Progesterone Through Integrated Bioinformatics Analysis
}

\section{OPEN ACCESS}

Edited by:

Udo Rudolf Markert,

University Hospital Jena, Germany

Reviewed by:

Meirong Du,

Fudan University, China

Federico Jensen,

University of Buenos Aires, Argentina

*Correspondence:

Yong Zeng

zengyong1966@gmail.com

Tailang Yin

reproductive@whu.edu.cn

Su LiU

sunnysue0309@163.com

${ }^{t}$ These authors have contributed equally to this work and share

the first authorship

Specialty section:

This article was submitted to Immunological Tolerance and Regulation,

a section of the journal

Frontiers in Immunology

Received: 31 October 2021 Accepted: 01 December 2021 Published: 17 December 2021

Citation:

Zhang S, Liu S, Hong L, Wang $X$, Diao L, Cai S, Yin T and Zeng Y (2021) Identification of Profound Metabolic Alterations in Human Dendritic Cells by

Progesterone Through Integrated Bioinformatics Analysis.

Front. Immunol. 12:806110.

doi: 10.3389/fimmu.2021.806110

\author{
Sainan Zhang ${ }^{1 \dagger}$, Su Liu ${ }^{2 *}$, Ling Hong ${ }^{2}$, Xiaohui Wang ${ }^{2}$, Lianghui Diao ${ }^{2}$, Songchen Cai ${ }^{2}$, \\ Tailang Yin ${ }^{1 *}$ and Yong Zeng ${ }^{2 *}$ \\ ${ }_{1}^{1}$ Reproductive Medical Center, Renmin Hospital of Wuhan University and Hubei Clinic Research Center for Assisted \\ Reproductive Technology and Embryonic Development, Wuhan, China, ${ }^{2}$ Shenzhen Key Laboratory of Reproductive \\ Immunology for Peri-implantation, Shenzhen Zhongshan Institute for Reproduction and Genetics, Shenzhen Zhongshan \\ Urology Hospital, Shenzhen, China
}

Maintaining the homeostasis of the decidual immune microenvironment at the maternalfetal interface is essential for reproductive success. Dendritic cells (DCs) are the professional antigen-presenting cells and dominate this balance of immunogenicity and tolerance. Progesterone (P4) is highlighted as the "hormone of pregnancy" in most eutherian mammals because of its regulatory role in immune-endocrine behavior during pregnancy. Recent studies have shown that P4 is associated with the differentiation and function of DCs, however, the underlying mechanisms remain unidentified. In addition, while progress in the field of immunometabolism has highlighted the intimate connections between the metabolism phenotype and the immunogenic or tolerogenic fate of DCs, whether P4 can affect DCs metabolism and thus exert a functional manipulation has not yet been explored. In this study, we acquired human peripheral blood monocyte-derived DCs and conducted RNA sequencing (RNA-seq) on immature DCs (iDCs), P4-treated DCs (pDCs), and mature DCs (mDCs), aiming to comprehensively assess the effects of P4 on DCs. Our results showed pDCs performed a distinct differentially expressed genes (DEGs) profile compared with iDCs or mDCs. Further functional enrichment and weighted gene co-expression network (WGCNA) analysis found that these DEGs were related not only to the cellular components but also to the significant metabolic activities, including mitochondrial oxidative phosphorylation (OXPHOS) and fatty acid metabolism. In addition, these changes may be involved in the activation of various signaling pathways of PI3K Akt/mTOR, AMPK/PGC1- $\alpha$, and PPAR- $\gamma$. In summary, our work suggested that P4 induced profound metabolic alterations of mitochondrial OXPHOS and fatty acid metabolism in DCs. Our findings may provide new insights into the role of P4 in DCs. 


\section{INTRODUCTION}

Pregnancy is a complex and highly coordinated event. Immunologically, the embryo is a semi-allograft that resides in an immune-competent mother, however, the distinct tolerable microenvironment at the maternal interface provides a guarantee for a successful pregnancy. The underlying mechanisms of maternal-fetal tolerance are multiple including the change of immune cells populations, induction of regulatory $\mathrm{T}$ cells, and the shift of Th1 pro-inflammatory to Th2 anti-inflammatory cytokine responses $(1,2)$. These alternations are sophisticatedly orchestrated under the immune-endocrine interactions. In particular, the female steroid hormone-progesterone (P4), is considered to occupy an important regulatory position in mediating immune response and maintaining pregnancy in humans (3).

P4 is mainly produced by the corpus luteum of the ovary in the menstrual cycle, and this function is subsequently taken over by the placenta after 8 weeks in pregnancy $(4,5)$. P4 is regarded as the "hormone of pregnancy" and its serum levels undergo a profound change ranging from $10^{-9} \mathrm{M}$ to $10^{-6} \mathrm{M}$ during pregnancy (6). P4 directly regulates gene transcription by binding specific receptors (7). Many studies have reported that P4 is widely involved in endometrial spiral artery remodeling, trophoblast cells adhesion, proliferation, and stromal cells decidualization $(8,9)$. In addition, studies have emphasized P4 as a communication bridge of endocrine-immune to mediate maternal tolerance to the fetus via acting on a series of decidual immune cells, including natural killer cells, T cells, and dendritic cells (DCs) (10-13). Remarkably, the effect of P4 on DCs has gained great attention because of its plastic talent in inducing antigen-specific immunity or tolerance at the maternalfetal interface.

DCs are originally described as the most potent antigenpresenting cell, and play a central role in both innate and adaptive immunity, despite their small proportions. DCs are divided into two different developmental stages: immature DCs (iDCs) and mature DCs (mDCs) (14). iDCs are usually found to have a limited migratory and $\mathrm{T}$ cell priming capacity and serve as "surveillance police" to induce immune tolerance, whereas mDCs exhibit an exceptional ability for antigen presentation and $\mathrm{T}$ cell activation and therefore triggers a strong immune response (15). Moreover, iDCs can differentiate toward immunogenic mDCs when activated by various antigens in vivo or typical toll-like receptor (TLR) agonists such as lipopolysaccharide (LPS) in vitro (16). DCs are not only positively regulated for their maturation as described above, but also negatively responded to signals that prevent their activation, such as dexamethasone and vitamin D $(17,18)$. Recently, some studies reported that P4 can suppress the production of pro-inflammatory cytokines TNF- $\alpha$ and IL- $1 \beta$, and reduce CD40 and CD80 expression in DCs (19-21). These studies emphasized that DCs were highly responsive to P4 and can be modified in terms of their activities and functions. However, the number of studies on this topic is still limited, and the underlying mechanism remains poorly understood.

Immunometabolism, an emerging field that explores how metabolism affects immune cell functions, has been well understood in the last decade $(22,23)$. Numerous studies have revealed that DCs performed preferred metabolism pathways in activated or resting status (24-26). Moreover, metabolism can also directly determine the immunogenicity or tolerability of DCs (27, 28). Interestingly, a recent study demonstrated that $\mathrm{P} 4$ can activate hypoxia-inducible factor $1 \alpha$ (HIF-1 $\alpha)$ and c-Myc signal to maintain aerobic glycolysis and decidualization in decidualized cells (29). Therefore, it is reasonable to speculate that the metabolism phenotype of DCs may be regulated by P4. In this study, we aimed to determine the metabolic changes and key modules genes underlying human peripheral blood monocytederived DCs treated with P4 (pDCs) by RNA sequencing (RNAseq) and weighted gene co-expression network analysis (WGCNA). Functional enrichment analysis was further applied to help in identifying the most important candidate genes involved in metabolism for quantitative real-time polymerase chain reaction (qRT-PCR) and immunoblot validation.

\section{MATERIALS \& METHODS}

\section{In Vitro Generation and Treatment of Human DCs}

This study was approved by the Institutional Review Board of Reproductive Research Ethics Committees of Shenzhen Zhongshan Urology Hospital (Approval number: SZZSECHU2020005). Informed consent was obtained following the Declaration of Helsinki. A protocol for the generation of human peripheral blood monocyte-derived DCs was based on previous research with some minor modifications (30). In brief, human monocytes were isolated from peripheral blood mononuclear cells (PBMCs) of healthy women volunteers by density centrifugation at $300 \mathrm{~g}$ for 10 minutes (Ficoll-Paque, GE Healthcare) and were purified by CD14 MicroBeads (Miltenyi Biotec, Bergisch Gladbach, Germany, purity $>95 \%$ ) according to the manufacturer's instructions. Then the cells were cultured in 24-well plates (Corning, China) with RPMI 1640 (Gibco, USA), supplemented with 10\% FBS (Gibco, USA), $100 \mathrm{U} / \mathrm{ml}$ penicillin, and $100 \mathrm{mg} / \mathrm{ml}$ streptomycin placed in an incubator under $5 \%$ $\mathrm{CO}_{2}$ at $37^{\circ} \mathrm{C} .10 \mathrm{ng} / \mathrm{mL}$ recombinant human IL-4 (R\&D Systems) and $20 \mathrm{ng} / \mathrm{mL}$ recombinant human granulocyte-macrophage colony-stimulating factor (GM-CSF) (R\&D Systems) were also added to induce the differentiation of the cells. Refreshment of the culture medium and cytokines was performed on day 2. To obtain P4-induced DCs, cells were treated on day 4 with $10^{-8} \mathrm{M}$ P4 (Sigma-Aldrich). Then cells were either treated with $1 \mu \mathrm{g} / \mathrm{mL}$ LPS (Sigma-Aldrich) on day 5 or left untreated. On day 7 , immature, mature, and P4-treated DCs were photographed with a High Content Analysis System (Operetta CLS, PerkinElmer) before being harvested for further experiments. The whole process was displayed in Figure 1A.

\section{RNA Collection and Sequencing}

Total RNA was isolated using RNA Purification Kit (Thermo Fisher) following the instructions of the manufacturer, and the purity and integrity were checked using the NanoPhotometer ${ }^{\circledR}$ spectrophotometer (IMPLEN, CA, USA) and RNA Nano 6000 

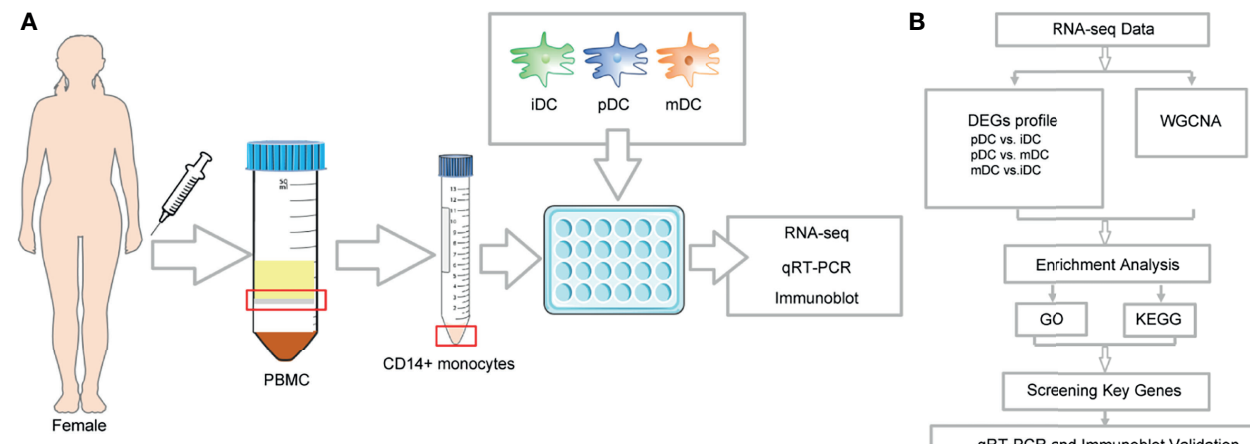

qRT-PCR and Immunoblot Validation

FIGURE 1 | Illustration of the study design framework and RNA-seq analysis flow.

Assay Kit of the Bioanalyzer 2100 system (Agilent Technologies, CA, USA), respectively. Subsequently, a total amount of $3 \mu \mathrm{g}$ RNA was used for the RNA sample preparations. First-strand and second-strand cDNA were synthesized according to the manufacturer of M-MuLV Reverse Transcriptase $\left(\mathrm{RNase} \mathrm{H}^{-}\right.$), and DNA Polymerase I and RNase H. cDNA fragments of 250 300 bp in length were selected and purified with the AMPure XP system (Beckman Coulter, Beverly, MA, USA). Then $3 \mu$ l USER Enzyme (NEB, USA) was used with cDNA at $37^{\circ} \mathrm{C}$ for $15 \mathrm{~min}$ followed by $5 \mathrm{~min}$ at $95^{\circ} \mathrm{C}$ before PCR and products were purified with AMPure XP system. Library quality was assessed on the Agilent Bioanalyzer 2100 system. The clustering of the index-coded samples was performed on a cBot Cluster Generation System using TruSeq PE Cluster Kit v3-cBot-HS (Illumia) according to the manufacturer's instructions. After cluster generation, the library preparations were sequenced on an Illumina Hiseq platform, and 125 bp/150 bp paired-end reads were generated. RNA-seq data have been deposited to NCBI's Sequence Read Archive with accession number PRJNA777391 (available at: https://www.ncbi.nlm.nih. gov/sra/). Finally, the overall workflow of data analysis was shown in Figure 1B.

\section{Differentially Expressed Genes (DEGs) Analysis}

The DESeq2 R package (1.16.1) was applied to perform DEGs analysis among iDCs, pDCs, and mDCs. The $P$-value was adjusted using Benjamini and Hochberg's approach for controlling the false discovery rate. Genes with an adjusted $P$-value of $<0.05$ were assigned as differentially expressed. The Venn diagram was drawn by using the online Venn diagrams tool (https://bioinfogp.cnb.csic.es/tools/venny/index.html) and volcano plots were represented with the R packages (1.16.1).

\section{Gene Ontology (GO) and Kyoto Encyclopedia of Genes and Genomes (KEGG) Enrichment Analysis}

GO and KEGG enrichment analysis of DEGs were implemented by the cluster profile $\mathrm{R}$ package. The GO terms consist of the following three parts: biological process (BP), cell component
(CC), and molecular function (MF). GO and KEGG terms with a corrected $P$-value of less than 0.05 were considered significantly enriched by DEGs.

\section{WGCNA Analysis}

WGCNA is a method for analyzing gene expression patterns of multiple samples, which can cluster genes with similar expression patterns and analyze the relationship between modules and specific traits or phenotypes. The genes with median absolute deviation $(\mathrm{MAD}) \leq 0.01$ were filtered, and the soft-power threshold of $\beta=13$ was selected. WGCNA analysis was performed in $\mathrm{R}$ with the WGCNA package. The further GO and KEGG enrichment was performed using Metascape.

\section{RNA Collection and qRT-PCR Analysis}

Total RNA was acquired as above described. 500 ng total RNA was used to synthesize cDNA using the PrimeScript ${ }^{\mathrm{TM}} \mathrm{RT}$ Reagent Kit with gDNA Eraser (Takara, Japan) with the BioRad system. Synthesized cDNA was carried for qRT-PCR in a 10 $\mu l$ reaction using Luna ${ }^{\circledR}$ Universal qPCR Master Mix (New England Biolabs) with primers specific to the genes. The reaction was performed in the QuantStudio 5 Real-Time PCR system (Applied Biosystems), following the manufacturer's instructions. The $2^{-\Delta \Delta} \mathrm{Ct}$ method was determined to calculate and quantify the gene expression. All mRNA levels were normalized to $\beta$-actin. Primers were designed with computer assistance based on GeneBank, and the sequences were listed in Table 1.

\section{Immunoblot Analysis}

Cells were harvested and lysed with radioimmunoprecipitation (RIPA; Beyotime, Shanghai, China) lysis buffer, and the lysates were centrifuged at $4^{\circ} \mathrm{C} 12000 \mathrm{~g}$ for $15 \mathrm{~min}$ to collect the supernatant. The BCA assay kit (Beyotime, Shanghai, China), was used to quantify protein concentrations. An automated Wes Capillary System (Protein Simple, San Jose, CA, 12-230 kDa kit cat. SM-W004) was used to detect the proteins levels of oxidative phosphorylation (OXPHOS) according to the manufacturer's protocol. Total protein loading was $0.75 \mu \mathrm{g} / \mu \mathrm{l}$. Antibody signals were determined to be saturated at 1:50 for Total OXPHOS 
TABLE 1 | Human forward and reverse primers for validation by qRT-PCR.

\begin{tabular}{|c|c|c|}
\hline Primers & Forward primer $\left(5^{\prime}-3^{\prime}\right)$ & Reverse primer $\left(5^{\prime}-3^{\prime}\right)$ \\
\hline MPC1 & ATाTGCCTACAAGGTACAGCC & AGTCATCTCGTGTITGATAAGCC \\
\hline $\mathrm{SDHC}$ & TAGGTTCAAACCGTCCTCTGT & GAGAGACCCCTGCACTCAAAG \\
\hline $\mathrm{SDHB}$ & TGAATAAGTGCGGACCTATGGTGTTG & GAGCCACAGATGCCTTCTCTACAAG \\
\hline UQCRQ & CGCGAGTTGGGAATCTGAC & TAGTGAAGACGTGCGGATAGG \\
\hline ATP5A & ATGACGACTTATCCAAACAGGC & CGGGAGTGTAGGTAGAACACAT \\
\hline COX II & ССАTCССТАCGСATCCTТТА & GTाTGCTCCACAGATITCAGAG \\
\hline NDUFB8 & ACAGGAACCGTGTGGATACAT & CCCCACCCAGCACATGAAT \\
\hline FASN & TCGTGGGCTACAGCATGGT & GCССTCTGAAGTCGAAGAAGAA \\
\hline ACACA & CATCAAATGCATCAGCAGAGACT & CTGCGTCATATGGATGATGGAAT \\
\hline HADHA & AAATTGACAGCGTATGCCATGA & GCTITCGCACTIITCTTCCACT \\
\hline HADHB & CTGTCCAGACCAAAACGAAGAA & CGATGCAACAAACCCGTAAGC \\
\hline СРT2 & CTGGAGCCAGAAGTGTTCCAC & AGGCACAAAGCGTATGAGTCT \\
\hline CPT1 & TCCAGTTGGCTTATCGTGGTG & TCCAGAGTCCGATTGATIITGC \\
\hline PI3K & AAGAAGCAAGCAGCTGAG & CTACAGAGCAGGCATAG \\
\hline AKT & GCCTITGCCGATCCGC & GCCGTAGCCGTTGTCG \\
\hline mTOR & ATGACGAGACCCAGGCTAA & GCCAGTCCTCTACAATACGC \\
\hline $\mathrm{HIF}-1 \alpha$ & GAAAGCGCAAGTCTTCAAAG & TGGGTAGGAGATGGAGATGC \\
\hline AMPK & GGCACGCCATACCCTTGAT & TCTTCCTTCGTACACGCAAATAA \\
\hline PGC1- $\alpha$ & TCTGAGTCTGTATGGAGTGACAT & CCAAGTCGTTCACATCTAGTTCA \\
\hline PPAR- $\gamma$ & CGGTGACTTATCCTGTGGTCC & CCGCAGATTCTACATTCGATGTT \\
\hline$\beta$-actin & TCGTGCGTGACATTAAGGAG & GTCAGGCAGCTCGTAGCTCT \\
\hline
\end{tabular}

MPC1, mitochondrial pyruvate carrier 1; SDHB, succinate dehydrogenase complex iron sulfur subunit B; SDHC, succinate dehydrogenase subunit C; UQCRQ, ubiquinol-cytochrome C

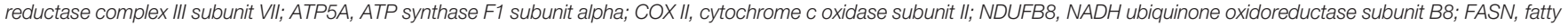

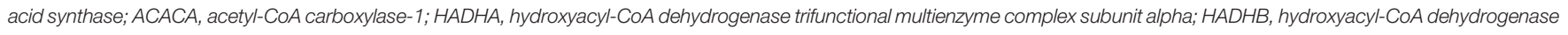
trifunctional multienzyme complex subunit beta; CPT2, carnitine palmitoyltransferase II; CPT1, carnitine palmitoyltransferase I; PI3K, phosphoinositide-3-kinase; AKT, protein kinase B;

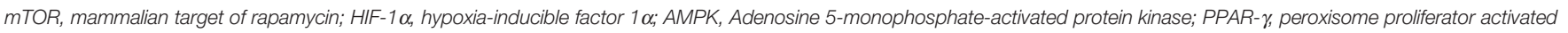
receptor gamma; PGC1- $\alpha$, PPAR- $\gamma$ coactivator 1 alpha; $q R T-P C R$, quantitative real-time polymerase chain reaction.

Human WB Antibody Cocktail (Abcam, ab110411) and 1:200 for vinculin antibody (Abcam, ab129002). Immunoblot analysis was executed using the Compass for Simple Western Program (Protein Simple).

\section{Statistical Analysis}

Continuous data with normal distribution were presented as the mean \pm standard error of mean (SEM), and analyzed by analysis of variance (ANOVA) using SPSS 22.0 (IBM SPSS, USA). Continuous variables without normal distribution were presented as median and interquartile range and were analyzed by Kruskal-Wallis test, pairwise comparison. All $P$-values were two-tailed and significance was set as ${ }^{\star} P$-value $<0.05$, ${ }^{* *}$ $P$-value $<0.01$. All experiments were independently repeated at least three times.

\section{RESULTS}

\section{Morphological Characterization of iDCs, pDCs, and $\mathrm{mDCs}$}

To investigate the alterations of human DCs exposed to P4, the morphological features of iDCs, pDCs, and mDCs were observed and photographed on day 7. Compared to the round-shaped iDCs, the mDCs showed more small dendrites, as previously reported (30). pDCs presented a completely different morphological feature from mDCs. These cells became larger with spindle-shaped dendrites (Figure 2). Moreover, all DCs appeared to be a typical characterization of the cell colony.
The result showed that DCs developed a distinct morphological change after P4 treatment.

\section{DEGs Identification of iDCs, pDCs, and $\mathrm{mDCs}$}

To investigate the effects of P4 on DCs, 5 samples in the iDCs, $\mathrm{pDCs}$, and $\mathrm{mDCs}$ group were selected for gene sequencing, respectively. Genes with an adjusted $P$-value $<0.05$ were categorized as differentially expressed. The overall distribution of DEGs was shown in a Venn diagram (Figure 3A). After normalization, P4 induced 846 genes alterations, with 314 upregulated genes and 532 downregulated genes compared with iDCs (Figure 3B). Comparatively, pDCs showed a total of 7289 DEGs, which contain 3054 upregulated and 4235 downregulated genes versus mDCs (Figure 3C). We also detected 6978 differential genes between iDCs and mDCs, with 3740 upregulated and 3238 downregulated genes (Figure 3D). These results revealed that P4-treated DCs behaved a distinct gene expression profile, especially when compared with mDCs.

\section{GO and KEGG Enrichment Analysis}

To recognize the feature and function of these DEGs in detail, we conducted GO and KEGG enrichment analysis by the cluster Profiler R package, in which gene length bias was corrected. GO terms with a corrected $P$-value of less than 0.05 were considered to be significantly enriched. Based on this screening criterion, we identified the most significant GO annotation, which consists of biological process (BP), cell composition (CC), and molecular function (MF). Compared to iDCs, DEGs in pDCs were mainly enriched in the regulation of cellular components size, 

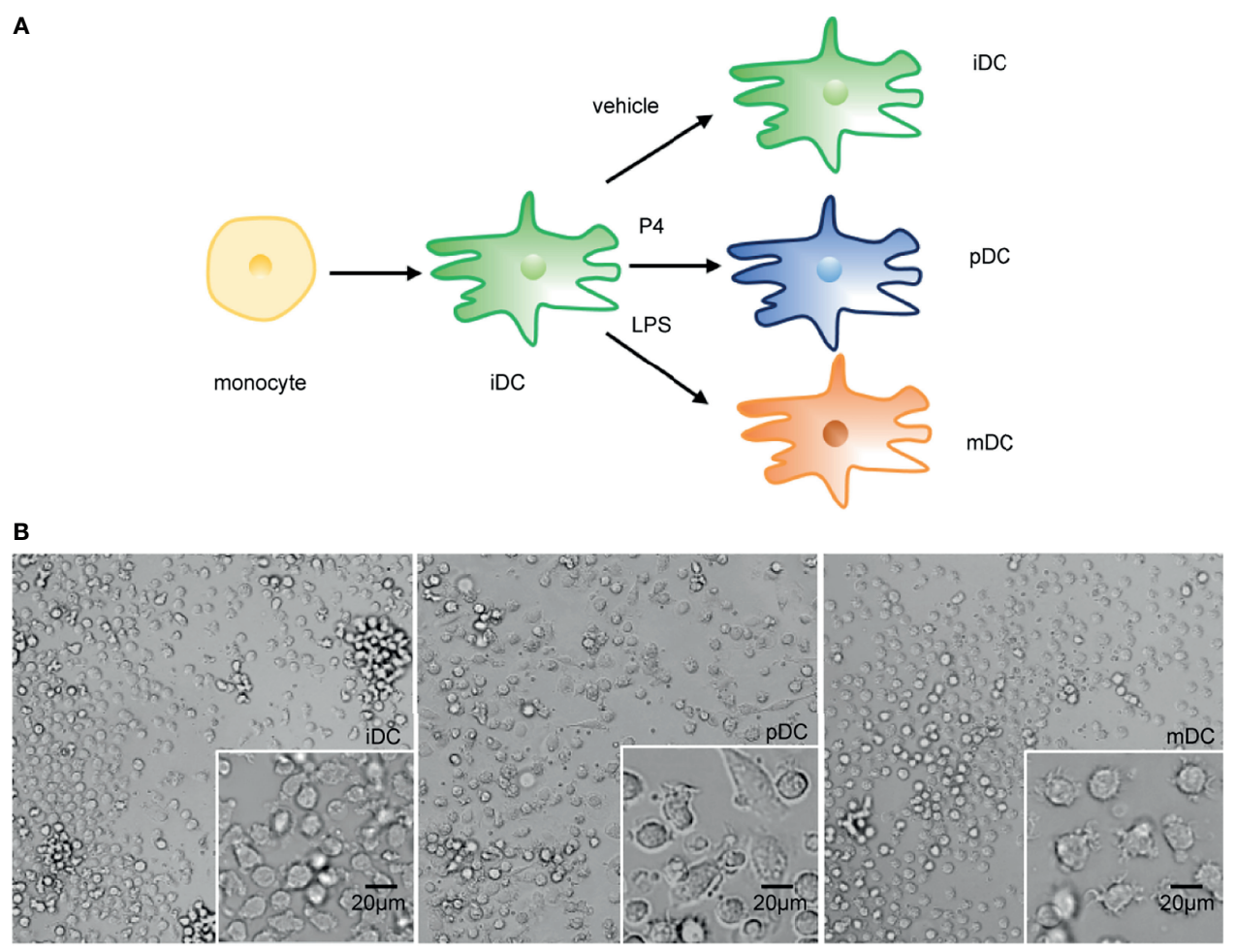

FIGURE 2 | Schematic diagram of DCs treatments and representative morphological pictures. (A) Schematic diagram of DCs treatments. (B) Representative monolayer cells pictures of iDC, pDC, and mDC on day 7 . Magnification, $\times 20 ; \times 40$. Scale bar $=20 \mu \mathrm{m}$. P4, progesterone; LPS, lipopolysaccharide; iDC, immature DC; pDC, P4-treated DC; mDC, mature DC.

cytoskeleton organization, actin-based cell projection (Figure 4A). When compared with mDCs, pDCs showed a significant gene enrichment associated with metabolic behaviors, including OXPHOS, mitochondrial protein complex, mitochondrial respiratory chain, and $\mathrm{NADH}$ dehydrogenase activity (Figure 4B). These GO terms were also illustrated as dot plots, with the gene ratio denoted by size, and the significance denoted by color (Figures 4C, D). The detailed gene information of the top $10 \mathrm{GO}$ annotations was also displayed in Table 2. To further investigate the potential pathway involved in these DEGs, we also selected the top 20 of KEGG enrichment results. We found that pDCs were mainly enriched in the regulation of actin cytoskeleton, phosphoinositide-3-kinase (PI3K)/protein kinase B (Akt) signaling, and glycerophospholipid metabolism compared with iDCs (Figure 4E). Interestingly, when compared with mDCs, pDCs presented significant enrichment involved in metabolic activities, including OXPHOS, citrate cycle, fatty acid elongation, and biosynthesis of unsaturated fatty acids (Figure 4F). We also listed the detailed gene information related to these metabolic pathways in Table 3. In addition, we observed several canonical metabolism-related signaling, including the peroxisome proliferator activated receptor (PPAR) signaling, mammalian target of rapamycin (mTOR) signaling, HIF- $1 \alpha$ signaling, and adenosine 5-monophosphate-activated protein kinase (AMPK) signaling although the $P$-values were slightly above the 0.05 significance threshold (Table S1). Collectively, these data implied that compared with iDCs or mDCs, the DEGs of pDCs were associated with the cellular cytoskeleton and cellular metabolic events including OXPHOS and fatty acid metabolism.

\section{WGCNA Analysis}

To further explore the changes that DCs respond to P4, we also conducted a WGCNA analysis. A scale-free co-expression network was constructed with a soft threshold of 13 (Figure 5A). Genes in the same module had similar expression patterns in different samples, and modules were distinguished by different colors. A total of 26 modules were generated, of which the blue, red, and green-yellow modules showed the highest correlation and were selected for further analysis (Figures 5B,C). Our enrichment results showed that $\mathrm{GO}$ annotations in the red module were mainly related to fatty acid metabolism, lipid biosynthetic process, acetyl-CoA, and $\mathrm{C}$-acyltransferase activity. The blue and green-yellow modules were enriched in mitochondrial biology, oxidoreductase activity, and the generation of cellular metabolites and energy (Figure 5D). Moreover, KEGG analysis of these three modules exhibited enrichment in metabolic activities, such as fatty acid elongation or oxidation (Figure 5E). These data suggested that P4 treatment induced the fatty acid metabolism and mitochondrial alterations of DCs. 

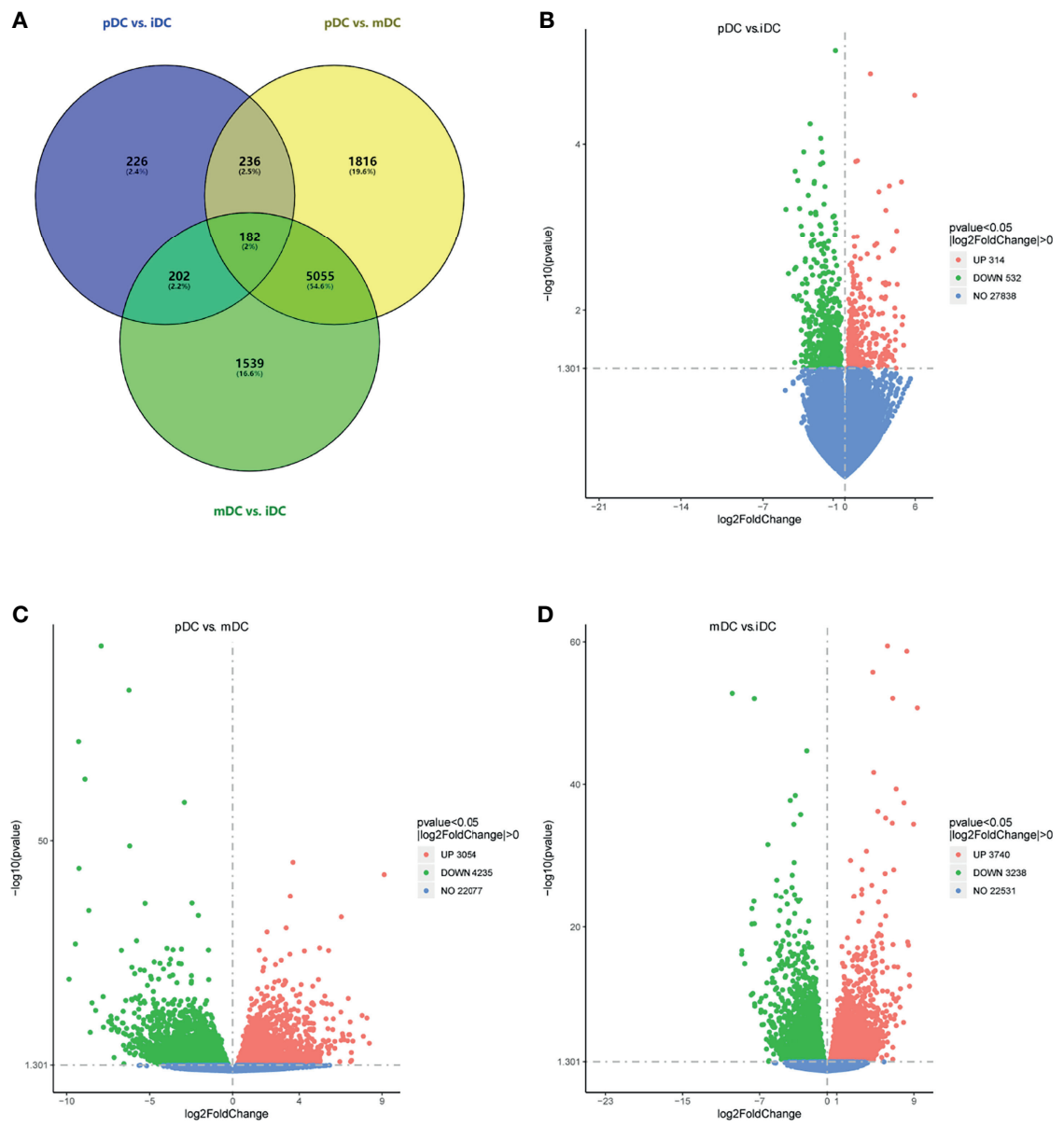

FIGURE 3 | DEGs analysis. (A) Venn diagram of DEGs (pDC vs. iDC, pDC vs. mDC, and mDC vs. iDC). (B-D) Volcano map of DEGs (pDC vs. iDC; $\mathrm{pDC}$ vs. mDC, and $\mathrm{mDC}$ vs. iDC, respectively). Red dots indicated upregulated DEGs, green dots indicated downregulated DEGs, and blue dots indicated genes that were not differentially expressed. DEGs, differentially expressed genes; iDC, immature DC; pDC, P4-treated DC; mDC, mature DC.

\section{Validation of qRT-PCR and Immunoblot}

To validate the RNA-seq data, we selected 20 genes related to the metabolism of all DEGs to perform qRT-PCR. Our results showed that the genes of mitochondrial complexes II-V (succinate dehydrogenase complex iron sulfur subunit $\mathrm{B}, \mathrm{SDHB}$; succinate dehydrogenase complex subunit $\mathrm{C}$, SDHC; ubiquinol-cytochrome C reductase complex III subunit VII, UQCRQ; cytochrome c oxidase subunit II, COX II, and ATP synthase F1 subunit alpha, ATP5A) were highly expressed in pDCs compared with mDCs, though no significant differences were detected in complex INADH ubiquinone oxidoreductase subunit B8 (NDUFB8) (Figure 6A). These complexes are important components of the electron transport chain and the oxygen-coupled ATP synthesis in the mitochondrial OXPHOS process. Our data showed that the mRNA levels of mitochondrial pyruvate carrier 1 (MPC1) were upregulated in pDCs compared with mDCs, which is a key factor for mitochondrial pyruvate carrier and sequent OXPHOS (Figure 6A). We also demonstrated significantly increased protein levels of OXPHOS complexes in pDCs compared with $\mathrm{mDCs}$ (Figures 6B, C). Additionally, we found that hydroxyacyl-
CoA dehydrogenase trifunctional multienzyme complex subunit beta (HADHB), carnitine palmitoyltransferase I (CPT1), and II (CPT2), which are necessary for fatty acid oxidation (FAO), and acetyl-CoA carboxylase alpha (ACACA) and fatty acid synthase (FASN), which are involved in the fatty acid synthesis (FAS), were also increased in mRNA levels in pDCs compared with mDCs (Figure 6A). Moreover, we observed upregulated mRNA expression of AMPK, PPAR- $\gamma$ coactivator 1 alpha (PGC1- $\alpha$ ), PI3K, Akt, mTOR, and PPAR- $\gamma$ in pDCs compared with mDCs, whereas the expression of HIF-1 $\alpha$ had no change (Figure 6A). Taken together, these findings suggested DCs present a distinct phenotype following $\mathrm{P} 4$ treatment with general trends in association with enhanced OXPHOS and fatty metabolic activities.

\section{DISCUSSION}

P4 has long been considered as the master hormone of pregnancy. In recent years, P4 is put forward as a potential medium for regulating the maternal immune system in vivo and 

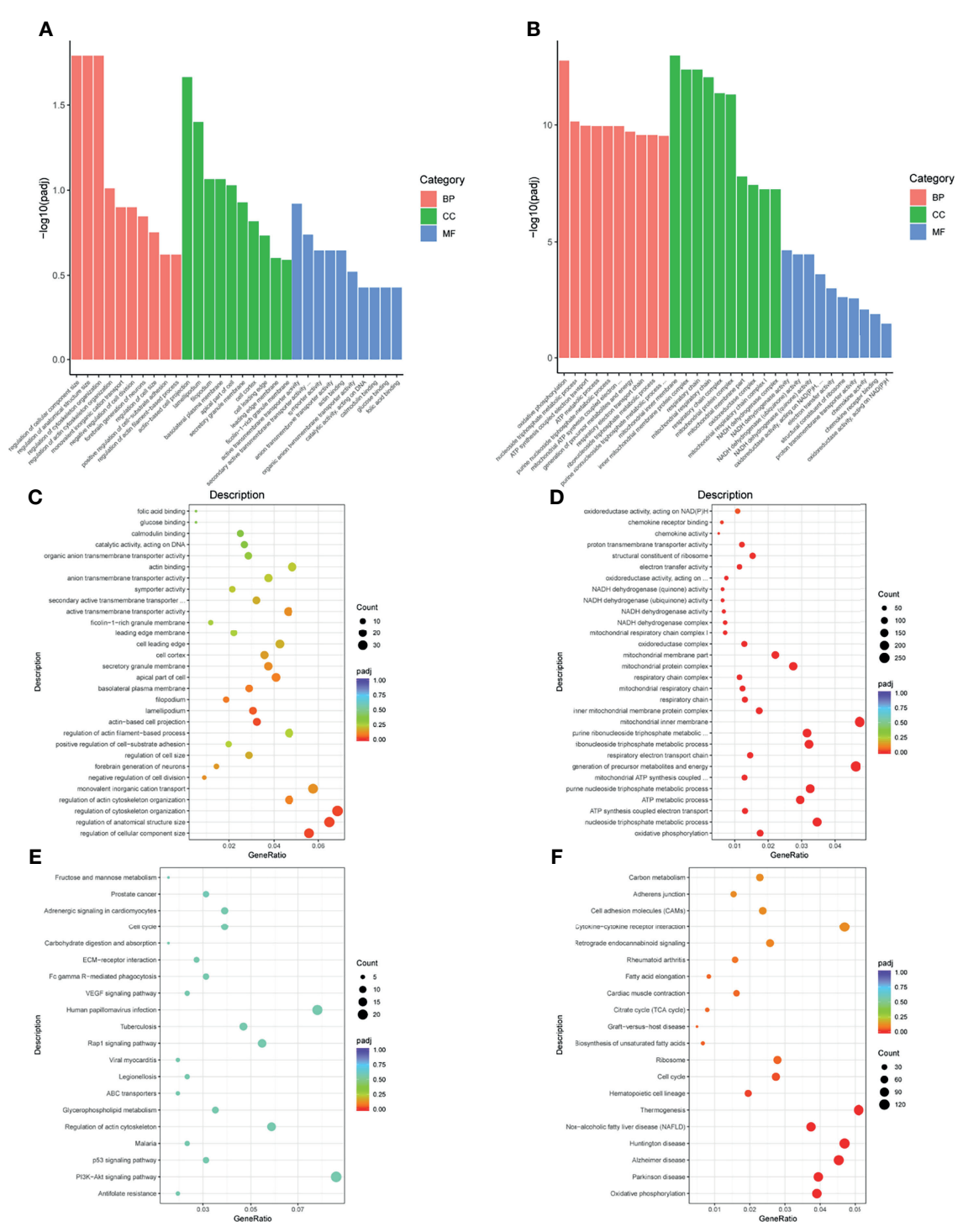

FIGURE 4 | GO and KEGG enrichment analysis. (A, B) Bars diagram of GO enrichment analysis (pDC vs. iDC and pDC vs. mDC, respectively). The X-axis denoted detailed annotation classes of $\mathrm{GO}$ ontologies by different colors and the $\mathrm{Y}$-axis denoted the percentage of genes. (C, D) Dots diagram of GO enrichment (pDC vs. iDC and pDC vs. $\mathrm{mDC}$, respectively). (E, F) Dots diagram of KEGG enrichment (pDC vs. iDC and pDC vs. mDC, respectively). The X-axis indicated the proportion of DEGs annotated to GO or KEGG terms to all GO or KEGG annotated DEGs and the Y-axis represented detailed classification of GO or KEGG. Size and color of dots represented the percentage of genes and significance level of adjusted P-value, respectively. GO, Gene Ontology; KEGG, Kyoto Encyclopedia of Genes and Genomes; BP, biological process; CC, cellular component; MF, molecular function; DEGs, differentially expressed genes; iDC, immature DC; pDC, P4-treated DC; $\mathrm{mDC}$, mature $\mathrm{DC}$.

for maintaining maternal-fetal immune tolerance $(13,31,32)$. These effects are achieved through interaction with a series of decidual immune cells, such as $\mathrm{T}$ cells, natural killer cells, macrophages, and DCs. Although some profound findings demonstrated the influence of $\mathrm{P} 4$ on lymphocytes, there are limited studies exploring the effects of P4 on DCs functions, and the mechanism underlying these effects $(19,33-35)$. DCs perform an extraordinary capacity to balance immunogenicity and tolerance for providing a protected environment during normal pregnancy. Therefore, it is interesting to explore the role of P4 on DCs. In the present study, we obtained an information- rich gene expression profile of human DCs treated with $\mathrm{P} 4$ by RNA-seq. Further analysis showed that P4 dramatically altered the expression of key genes and pathways involved in the metabolism of DCs, which may be a promising manipulator in modulating DCs tolerance. This current study provided the first evidence that $\mathrm{P} 4$ regulated the metabolism of DCs and helped to further improve our understanding of the effect of P4 on DCs.

In this study, we firstly analyzed the treatment and the corresponding gene data of DCs and found pDCs behaved a significant different DEGs profile from that of iDCs or mDCs. Compared to iDCs, the GO enrichment analysis in pDCs 
TABLE 2 | Top 10 enriched GO terms.

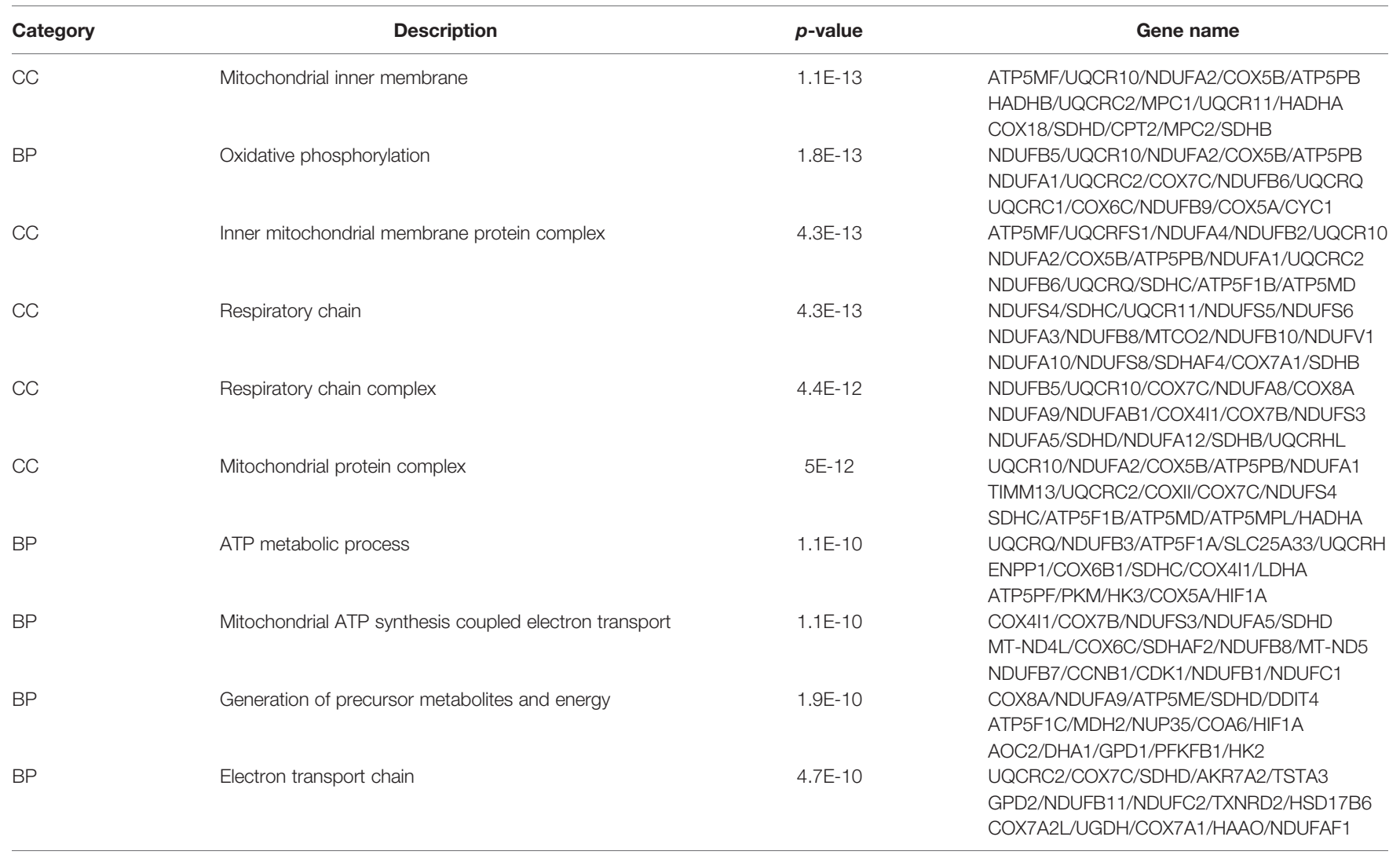

GO, Gene Ontology; BP, biological process; CC, cellular component; MF, molecular function.

TABLE 3 | Most significant KEGG metabolism pathways.

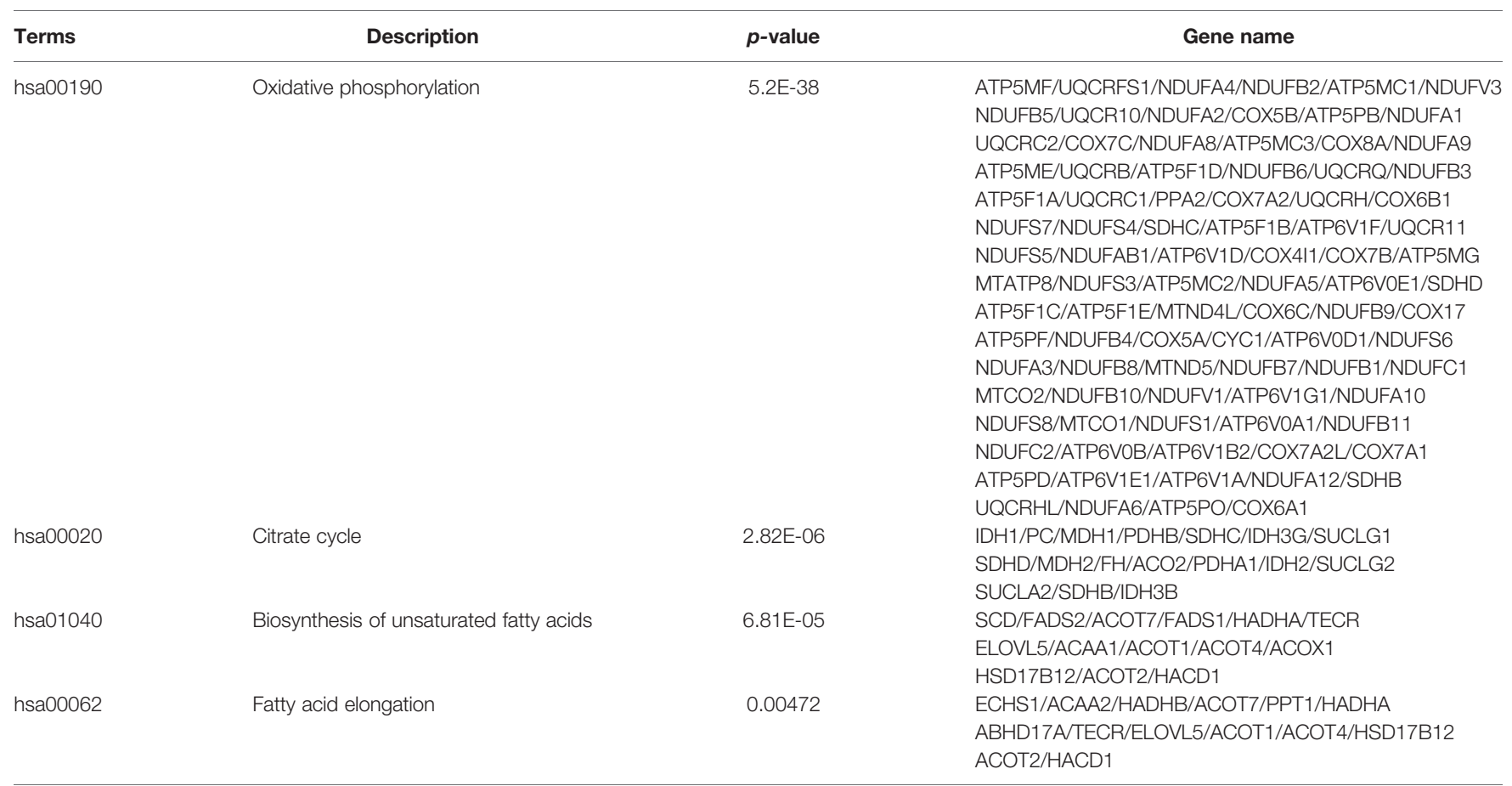

KEGG, Kyoto Encyclopedia of Genes and Genomes. 


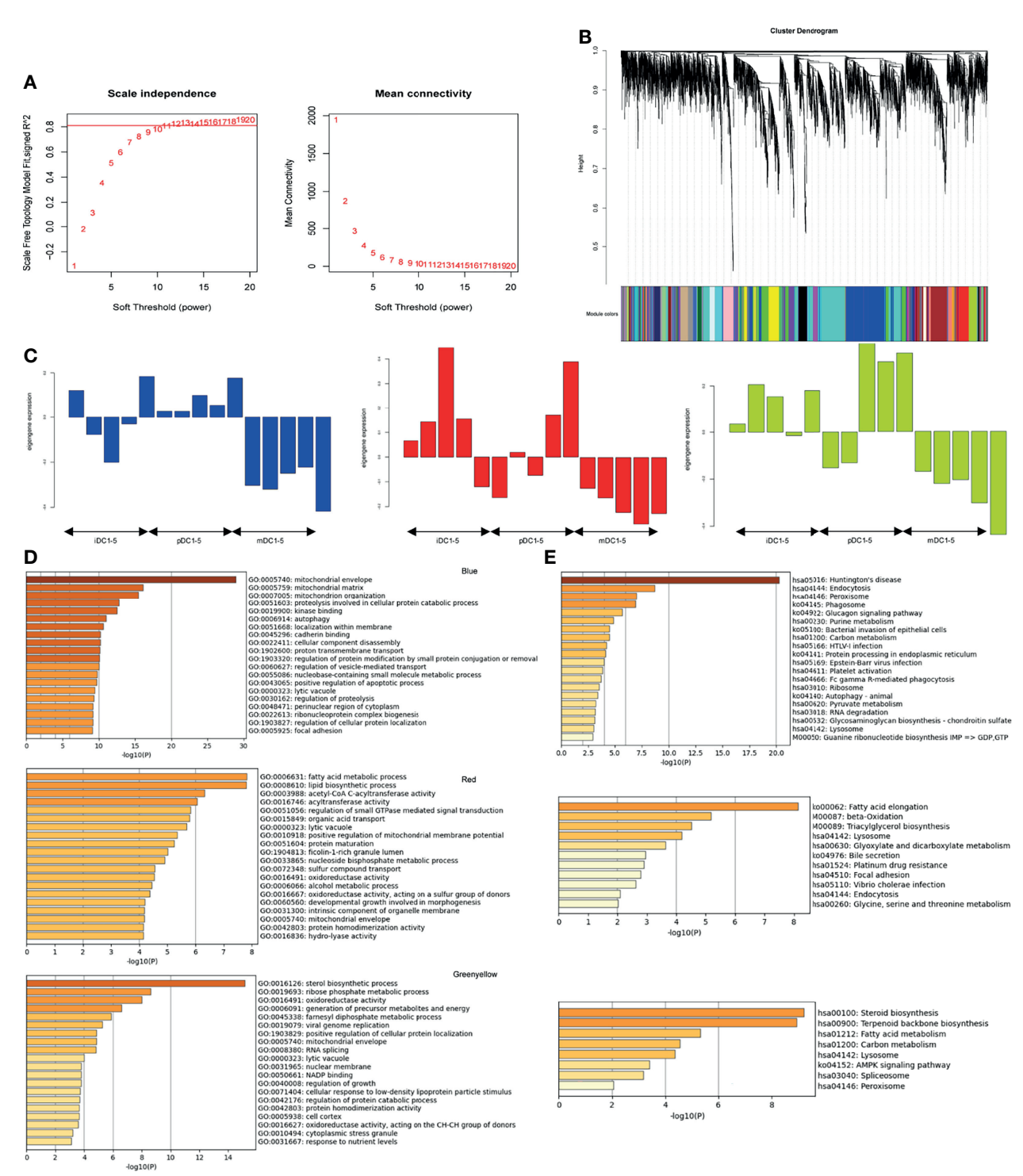

FIGURE 5 | WGCNA analysis. (A) Determination of soft-threshold power. (B) Cluster dendrogram of genes. (C) Expression pattern of the genes and eigengenes in the blue, red, and green-yellow modules. (D) GO enrichment results for genes in blue, red, and green-yellow modules. (E) KEGG enrichment results for genes in blue, red, and green-yellow modules. GO, Gene Ontology; KEGG, Kyoto encyclopedia of genes and genomes; iDC, immature DC; pDC, P4-treated DC; mDC, mature DC.

revealed enriched terms related to cellular components and cytoskeleton. Interestingly, the mitochondria were identified as the core of GO cellular component terms in pDCs, such as mitochondrial protein complex, mitochondrial inner membrane, mitochondrial respiratory chain, and electron transfer activity compared with mDCs. The mitochondrial respiratory chain, also known as the mitochondrial electron transport chain, consists of five enzyme complexes (complexes I-V) situated in the inner mitochondrial membrane that couple oxidation to phosphorylation, and provide ATP. KEGG analysis also showed that DEGs in pDCs were primarily concentrated in the OXPHOS process and fatty acid metabolism. These data suggested that $\mathrm{P} 4$ induced obvious changes in gene transcription levels that point to mitochondrial metabolism of DCs. Our qRT-PCR validation demonstrated that P4 upregulated the mRNA levels of mitochondrial complexes II-V (SDHB, SDHC, UQCRQ, ATP5A, COX II) in DCs. We also further confirmed increased protein levels of OXPHOS complexes, including SDHB, COX II, and ATP5A. In addition, we found upregulated mRNA levels of MPC1, which is essential for mitochondrial pyruvate transport and subsequent OXPHOS process. These findings together indicated that human DCs initiated early OXPHOS metabolic pathways at the mRNA and protein levels after exposure to P4. According to WGCNA results and literature research, we also selected genes related to $\mathrm{FAO}$ (CPT1, CPT2, HADHB) and FAS (FASN, ACACA) for 

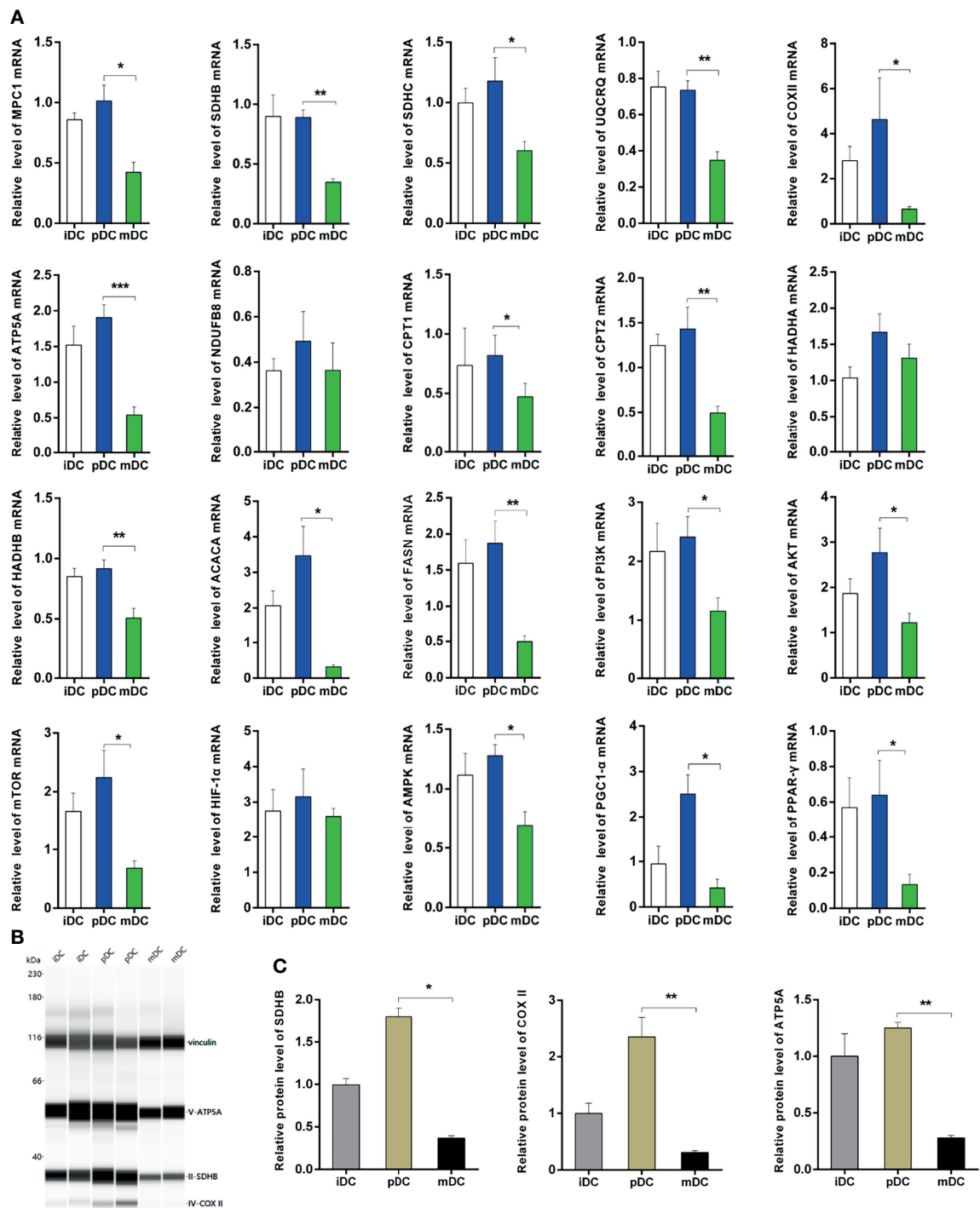

FIGURE 6 Validation of qRT-PCR and immunoblot. (A) Expression of genes associated with OXPHOS, FAO, and FAS using qRT-PCR. All values were normalized against the mRNA expression level of $\beta$-actin. (B, C) Protein levels and quantification of OXPHOS. qRT-PCR, quantitative real-time polymerase chain reaction; OXPHOS, oxidative phosphorylation; FAO, fatty acid oxidation; FAS, fatty acid synthesis; iDC, immature DC; pDC, P4-treated DC; mDC, mature DC. ${ }^{\star} \mathrm{P}<0.05$, ${ }^{\star \star} \mathrm{P}<$ $0.01,{ }^{* *} \mathrm{P}<0.001$.

validation, and the results showed that these genes were significantly upregulated in pDCs compared with mDCs. In detail, FAO involves two key steps of fatty acid transfer and $\beta$ oxidation. Both CPT1 and CPT2 are crucial to transfer activated long-chain fatty acids into mitochondria while HADHB acts in the subsequent $\beta$-oxidation process as one of the mitochondrial thiolates. In summary, our study identified profound mitochondrial and fatty metabolic alterations in human DCs regulated by $\mathrm{P} 4$.
It has long been acknowledged that the activation and function of immune cells correlate with, and are supported by alterations in their metabolic pathways $(22,23)$. Phenomena accompanying immunological responses involve changes in gene expression that are thermodynamically demanding, requiring fast metabolic adaptations, which is known as immunometabolism. Accumulating evidence has indicated that the metabolic programming of immune cells is an important determinant of their function $(36,37)$. DCs were reported to undergo metabolic 
reprogramming in response to multiple environmental factors. To date, the metabolic features of immunogenic DCs have become more well characterized. Krawczyk et al. demonstrated that TLR agonists stimulated a profound metabolic transition to aerobic glycolysis in mouse DCs. This metabolic switch depended on the PI3K/Akt pathway, was antagonized by the AMPK, and was important for DCs maturation and function (38). Induction of DCs glycolysis was also essential for the anabolic demands that underpinned the production of membranes needed for expansion of the endoplasmic reticulum and Golgi to accommodate the stimulation via TLR agonists (39). Several studies have reported metabolic characteristics of tolerogenic DCs despite the limitations of the available research. Proteomic analysis of human DCs treated with vitamin D and dexamethasone, two well-known immunosuppressive agents that induced tolerogenic DCs, revealed increased expression of genes associated with mitochondrial metabolism and OXPHOS $(17,30)$. Malinarich et al. confirmed similar results that human tolerogenic DCs displayed a markedly augmented catabolic pathway, related to high oxidative phosphorylation and fatty acid metabolism levels (40). Another recent study showed that tolerogenic effects of vitamin D on DCs also involved FAS (18). Thus, a current generally accepted opinion shows that increased OXPHOS and FAO are tightly associated with the tolerogenic function of DCs $(25,28,41,42)$. The above findings emphasize that the activation and function of DCs are dictated by the type of metabolism these cells commit to. In our current study, P4-treated DCs performed high OXPHOS and fatty acid metabolism capacities. This may provide promising information that $\mathrm{P} 4$ may be another potential tolerogenic medium that coordinates the metabolism and immune tolerogenic function in DCs during pregnancy. These observations also point to a mechanism for rapid genome-wide reprogramming by modulation of underlying cellular metabolism during DC differentiation. Our findings may contribute to the identification of $\mathrm{P} 4$ as new metabolic targets for manipulating DC function. This knowledge may be used in the rational design of strategies to improve the immunogenicity or tolerogenic of DCs in clinically relevant settings.

Additionally, we also tentatively demonstrated that P4 upregulated mRNA levels of PI3K, Akt, mTOR, AMPK, PGC1- $\alpha$, and PPAR- $\gamma$ in DCs. The mTOR and its upstream PI3K/Akt have been well known as metabolism-related regulation signaling pathways in DCs (24). AMPK is a central regulator of catabolic metabolism and has been shown to activate the PGC-1 $\alpha$, which promotes mitochondrial biogenesis to increase mitochondrial OXPHOS (43). PPAR- $\gamma$, a sensor for fatty acids, was recently confirmed to be involved in the functional regulation of human DCs (27). Taken together, these data suggested that the above metabolic effects in pDCs may be mediated by these signaling pathways, however, further studies are needed to clarify the mechanism.

In summary, our current study revealed a comprehensive RNA-seq analysis and identified some critical molecules and pathways related to metabolism between P4 and DCs. Our results provided the first evidence that P4-treated DCs had a defined metabolic phenotype characterized by a prominent mitochondrial OXPHOS and high fatty acid metabolic phenotype. These data may provide a novel insight into the role of $\mathrm{P} 4$ in DCs.

\section{DATA AVAILABILITY STATEMENT}

The datasets presented in this study can be found in online repositories. The names of the repository/repositories and accession number(s) can be found below: https://www.ncbi. nlm.nih.gov/, accession ID: PRJNA777391.

\section{ETHICS STATEMENT}

The studies involving human participants were reviewed and approved by The Institutional Review Board of Reproductive Research Ethics Committees of Shenzhen Zhongshan Urology Hospital. The patients/participants provided their written informed consent to participate in this study.

\section{AUTHOR CONTRIBUTIONS}

YZ, TY, and SL supported the research. SZ and SL conceived the original idea and the structure of the manuscript. SZ performed the experiments and drafted the first version of the manuscript. LH assisted in the experiments and manuscript. XW collected the clinical samples. LD and SC provided critical feedback and helped revise the manuscript. All authors contributed to the article and approved the submitted version.

\section{FUNDING}

This work was supported by the National Key Research \& Developmental Program of China (2018YFC1003900/ 2018YFC1003904/2018YFC1004601), National Natural Science Foundation of China (No. 82001547), Guangdong Basic and Applied Basic Research Foundation (2019A1515010914), Shenzhen Fundamental Research Program (JCYJ20190813160809117).

\section{ACKNOWLEDGMENTS}

The authors are very grateful to the staff at the Fertility Center at Shenzhen Zhongshan Urology Hospital for recruiting patients and helping with samples.

\section{SUPPLEMENTARY MATERIAL}

The Supplementary Material for this article can be found online at: https://www.frontiersin.org/articles/10.3389/fimmu.2021.806110/ full\#supplementary-material 


\section{REFERENCES}

1. La Rocca C, Carbone F, Longobardi S, Matarese G. The Immunology of Pregnancy: Regulatory T Cells Control Maternal Immune Tolerance Toward the Fetus. Immunol Lett (2014) 162(1 Pt A):41-8. doi: 10.1016/j.imlet. 2014.06.013

2. PrabhuDas M, Bonney E, Caron K, Dey S, Erlebacher A, Fazleabas A, et al. Immune Mechanisms at the Maternal-Fetal Interface: Perspectives and Challenges. Nat Immunol (2015) 16(4):328-34. doi: 10.1038/ni.3131

3. Piccinni MP, Raghupathy R, Saito S, Szekeres-Bartho J. Cytokines, Hormones and Cellular Regulatory Mechanisms Favoring Successful Reproduction. Front Immunol (2021) 12:717808. doi: 10.3389/fimmu.2021.717808

4. Stocco C, Telleria C, Gibori G. The Molecular Control of Corpus Luteum Formation, Function, and Regression. Endocr Rev (2007) 28(1):117-49. doi: 10.1210/er.2006-0022

5. Arck P, Hansen PJ, Mulac Jericevic B, Piccinni MP, Szekeres-Bartho J. Progesterone During Pregnancy: Endocrine-Immune Cross Talk in Mammalian Species and the Role of Stress. Am J Reprod Immunol (2007) 58(3):268-79. doi: 10.1111/j.1600-0897.2007.00512.x

6. Lydon JP, DeMayo FJ, Funk CR, Mani SK, Hughes AR, Montgomery CAJr., et al. Mice Lacking Progesterone Receptor Exhibit Pleiotropic Reproductive Abnormalities. Genes Dev (1995) 9(18):2266-78. doi: 10.1101/gad.9.18.2266

7. Szekeres-Bartho J, Halasz M, Palkovics T. Progesterone in Pregnancy; Receptor-Ligand Interaction and Signaling Pathways. J Reprod Immunol (2009) 83(1-2):60-4. doi: 10.1016/j.jri.2009.06.262

8. Kim M, Park HJ, Seol JW, Jang JY, Cho YS, Kim KR, et al. VEGF-A Regulated by Progesterone Governs Uterine Angiogenesis and Vascular Remodelling During Pregnancy. EMBO Mol Med (2013) 5(9):1415-30. doi: 10.1002/ emmm.201302618

9. Wang J, Liu S, Qin HM, Zhao Y, Wang XQ, Yan Q. Pregnancy-Associated Plasma Protein A Up-Regulated by Progesterone Promotes Adhesion and Proliferation of Trophoblastic Cells. Int J Clin Exp Pathol (2014) 7(4):1427-37.

10. Stites DP, Siiteri PK. Steroids as Immunosuppressants in Pregnancy. Immunol $\operatorname{Rev}$ (1983) 75:117-38. doi: 10.1111/j.1600-065x.1983.tb01093.x

11. Miyaura H, Iwata M. Direct and Indirect Inhibition of Th1 Development by Progesterone and Glucocorticoids. J Immunol (2002) 168(3):1087-94. doi: 10.4049/jimmunol.168.3.1087

12. Siiteri PK, Stites DP. Immunologic and Endocrine Interrelationships in Pregnancy. Biol Reprod (1982) 26(1):1-14. doi: 10.1095/biolreprod26.1.1

13. Shah NM, Imami N, Johnson MR. Progesterone Modulation of PregnancyRelated Immune Responses. Front Immunol (2018) 9:1293. doi: 10.3389/ fimmu.2018.01293

14. O’Doherty U, Peng M, Gezelter S, Swiggard WJ, Betjes M, Bhardwaj N, et al. Human Blood Contains Two Subsets of Dendritic Cells, One Immunologically Mature and the Other Immature. Immunology (1994) 82(3):487-93.

15. Hopkins RA, Connolly JE. The Specialized Roles of Immature and Mature Dendritic Cells in Antigen Cross-Presentation. Immunol Res (2012) 53(13):91-107. doi: 10.1007/s12026-012-8300-z

16. Reis e Sousa C. Dendritic Cells in a Mature Age. Nat Rev Immunol (2006) 6 (6):476-83. doi: 10.1038/nri1845

17. Ferreira GB, Kleijwegt FS, Waelkens E, Lage K, Nikolic T, Hansen DA, et al. Differential Protein Pathways in 1,25-Dihydroxyvitamin $\mathrm{D}(3)$ and Dexamethasone Modulated Tolerogenic Human Dendritic Cells. J Proteome Res (2012) 11(2):941-71. doi: 10.1021/pr200724e

18. Garcia AM, Bishop EL, Li D, Jeffery LE, Garten A, Thakker A, et al. Tolerogenic Effects of 1,25-Dihydroxyvitamin D on Dendritic Cells Involve Induction of Fatty Acid Synthesis. J Steroid Biochem Mol Biol (2021) 211:105891. doi: 10.1016/j.jsbmb.2021.105891

19. Quispe Calla NE, Ghonime MG, Cherpes TL, Vicetti Miguel RD. Medroxyprogesterone Acetate Impairs Human Dendritic Cell Activation and Function. Hum Reprod (2015) 30(5):1169-77. doi: 10.1093/humrep/ $\operatorname{dev} 035$

20. Mackern-Oberti JP, Jara EL, Riedel CA, Kalergis AM. Hormonal Modulation of Dendritic Cells Differentiation, Maturation and Function: Implications for the Initiation and Progress of Systemic Autoimmunity. Arch Immunol Ther Exp (Warsz) (2017) 65(2):123-36. doi: 10.1007/s00005-016-0418-6
21. Butts CL, Shukair SA, Duncan KM, Bowers E, Horn C, Belyavskaya E, et al. Progesterone Inhibits Mature Rat Dendritic Cells in a Receptor-Mediated Fashion. Int Immunol (2007) 19(3):287-96. doi: 10.1093/intimm/dxl145

22. O'Neill LA, Kishton RJ, Rathmell J. A Guide to Immunometabolism for Immunologists. Nat Rev Immunol (2016) 16(9):553-65. doi: 10.1038/ nri.2016.70

23. Ganeshan K, Chawla A. Metabolic Regulation of Immune Responses. Annu Rev Immunol (2014) 32:609-34. doi: 10.1146/annurev-immunol-032713120236

24. Pearce EJ, Everts B. Dendritic Cell Metabolism. Nat Rev Immunol (2015) 15 (1):18-29. doi: $10.1038 /$ nri3771

25. O'Neill LA, Pearce EJ. Immunometabolism Governs Dendritic Cell and Macrophage Function. J Exp Med (2016) 213(1):15-23. doi: 10.1084/jem. 20151570

26. He Z, Zhu X, Shi Z, Wu T, Wu L. Metabolic Regulation of Dendritic Cell Differentiation. Front Immunol (2019) 10:410. doi: 10.3389/fimmu. 2019.00410

27. Szatmari I, Töröcsik D, Agostini M, Nagy T, Gurnell M, Barta E, et al. PPARgamma Regulates the Function of Human Dendritic Cells Primarily by Altering Lipid Metabolism. Blood (2007) 110(9):3271-80. doi: 10.1182/blood2007-06-096222

28. Sim WJ, Ahl PJ, Connolly JE. Metabolism Is Central to Tolerogenic Dendritic Cell Function. Mediators Inflamm (2016) 2016:2636701. doi: 10.1155/2016/ 2636701

29. Zuo RJ, Gu XW, Qi QR, Wang TS, Zhao XY, Liu JL, et al. Warburg-Like Glycolysis and Lactate Shuttle in Mouse Decidua During Early Pregnancy. J Biol Chem (2015) 290(35):21280-91. doi: 10.1074/jbc.M115.656629

30. Ferreira GB, van Etten E, Lage K, Hansen DA, Moreau Y, Workman CT, et al. Proteome Analysis Demonstrates Profound Alterations in Human Dendritic Cell Nature by TX527, an Analogue of Vitamin D. Proteomics (2009) 9 (14):3752-64. doi: 10.1002/pmic.200800848

31. Szekeres-Bartho J. The Role of Progesterone in Feto-Maternal Immunological Cross Talk. Med Princ Pract (2018) 27(4):301-7. doi: 10.1159/000491576

32. Lissauer D, Eldershaw SA, Inman CF, Coomarasamy A, Moss PA, Kilby MD. Progesterone Promotes Maternal-Fetal Tolerance by Reducing Human Maternal T-Cell Polyfunctionality and Inducing a Specific Cytokine Profile. Eur J Immunol (2015) 45(10):2858-72. doi: 10.1002/eji.201445404

33. Xiu F, Anipindi VC, Nguyen PV, Boudreau J, Liang H, Wan Y, et al. High Physiological Concentrations of Progesterone Reverse Estradiol-Mediated Changes in Differentiation and Functions of Bone Marrow Derived Dendritic Cells. PloS One (2016) 11(4):e0153304. doi: 10.1371/journal. pone. 0153304

34. Jones LA, Kreem S, Shweash M, Paul A, Alexander J, Roberts CW. Differential Modulation of TLR3- and TLR4-Mediated Dendritic Cell Maturation and Function by Progesterone. J Immunol (2010) 185(8):4525-34. doi: 10.4049/ jimmunol.0901155

35. Butts CL, Candando KM, Warfel J, Belyavskaya E, D’Agnillo F, Sternberg EM. Progesterone Regulation of Uterine Dendritic Cell Function in Rodents Is Dependent on the Stage of Estrous Cycle. Mucosal Immunol (2010) 3(5):496505. doi: $10.1038 / \mathrm{mi} .2010 .28$

36. DeBerardinis RJ, Lum JJ, Hatzivassiliou G, Thompson CB. The Biology of Cancer: Metabolic Reprogramming Fuels Cell Growth and Proliferation. Cell Metab (2008) 7(1):11-20. doi: 10.1016/j.cmet.2007.10.002

37. Donnelly RP, Loftus RM, Keating SE, Liou KT, Biron CA, Gardiner CM, et al. Mtorc1-Dependent Metabolic Reprogramming Is a Prerequisite for NK Cell Effector Function. J Immunol (2014) 193(9):4477-84. doi: 10.4049/ jimmunol.1401558

38. Krawczyk CM, Holowka T, Sun J, Blagih J, Amiel E, DeBerardinis RJ, et al. Toll-Like Receptor-Induced Changes in Glycolytic Metabolism Regulate Dendritic Cell Activation. Blood (2010) 115(23):4742-9. doi: 10.1182/blood2009-10-249540

39. Everts B, Amiel E, Huang SC, Smith AM, Chang CH, Lam WY, et al. TLRDriven Early Glycolytic Reprogramming via the Kinases TBK1-IKKe Supports the Anabolic Demands of Dendritic Cell Activation. Nat Immunol (2014) 15(4):323-32. doi: 10.1038/ni.2833

40. Malinarich F, Duan K, Hamid RA, Bijin A, Lin WX, Poidinger M, et al. High Mitochondrial Respiration and Glycolytic Capacity Represent a Metabolic 
Phenotype of Human Tolerogenic Dendritic Cells. J Immunol (2015) 194 (11):5174-86. doi: 10.4049/jimmunol.1303316

41. Everts B, Pearce EJ. Metabolic Control of Dendritic Cell Activation and Function: Recent Advances and Clinical Implications. Front Immunol (2014) 5:203. doi: 10.3389/fimmu.2014.00203

42. Zhuang Q, Cai H, Cao Q, Li Z, Liu S, Ming Y. Tolerogenic Dendritic Cells: The Pearl of Immunotherapy in Organ Transplantation. Front Immunol (2020) 11:552988. doi: 10.3389/fimmu.2020.552988

43. Hardie DG. AMP-Activated/SNF1 Protein Kinases: Conserved Guardians of Cellular Energy. Nat Rev Mol Cell Biol (2007) 8(10):774-85. doi: 10.1038/ nrm 2249

Conflict of Interest: The authors declare that the research was conducted in the absence of any commercial or financial relationships that could be construed as a potential conflict of interest.
The handling Editor declared a past co-authorship with two of the authors LD and YZ.

Publisher's Note: All claims expressed in this article are solely those of the authors and do not necessarily represent those of their affiliated organizations, or those of the publisher, the editors and the reviewers. Any product that may be evaluated in this article, or claim that may be made by its manufacturer, is not guaranteed or endorsed by the publisher.

Copyright $\odot 2021$ Zhang, Liu, Hong, Wang, Diao, Cai, Yin and Zeng. This is an openaccess article distributed under the terms of the Creative Commons Attribution License (CCBY). The use, distribution or reproduction in other forums is permitted, provided the original author(s) and the copyright owner(s) are credited and that the original publication in this journal is cited, in accordance with accepted academic practice. No use, distribution or reproduction is permitted which does not comply with these terms. 